\title{
The unique association between the level of peripheral blood monocytes and the prevalence of diabetic retinopathy: a cross-sectional study
}

Heng Wan ${ }^{1 \dagger}$, Yan Cai ${ }^{2 \dagger}$, Yuying Wang ${ }^{1 \dagger}$, Sijie Fang ${ }^{3}$, Chi Chen ${ }^{1}$, Yi Chen ${ }^{1}$, Fangzhen Xia ${ }^{1}$, Ningjian Wang ${ }^{1 *}$, Minghao Guo ${ }^{1^{*}}$ and Yingli $\mathrm{Lu}^{1^{*}}$ (D)

\begin{abstract}
Objective: The attraction and influx of monocytes into the retina has been considered a critical step in the development of diabetic retinopathy (DR). However, large population studies about the association between peripheral blood monocyte levels, an inexpensive and easily measurable laboratory index, and DR are limited. Thus, we aimed to investigate the association between peripheral blood monocyte levels and DR.

Methods: A total of 3223 participants out of 3277 adults with diabetes were enrolled from seven communities in China in this cross-sectional survey. Participants underwent several medical examinations, including the measurement of anthropometric factors, blood pressure, routinely analyzed leukocyte characteristics, glucose, lipid profiles, urine albumin/creatinine ratio and fundus photographs.

Results: The prevalence of DR among the participants in the highest quartile of peripheral blood monocyte levels significantly decreased by $41 \%(\mathrm{OR} 0.59 ; 95 \% \mathrm{Cl} 0.43,0.81)$ compared with the participants in the first quartile ( $P$ for trend $<0.05)$. However, there were no associations between the monocyte level and the prevalence of cardiovascular and cerebrovascular diseases (CVD) and diabetic kidney disease (DKD) (both $P$ for trend $>0.05$ ). Associations between leukocyte, neutrophil and lymphocyte levels and DR were also not found (all $P$ for trend $>0.05$ ). These associations were all fully adjusted for age, sex, education status, duration of diabetes history, current smoking, BMI, HbA1c, dyslipidemia, systolic blood pressure and insulin therapy.

Conclusion: Decreased peripheral blood monocyte levels were associated with increased odds of DR after adjusting for potential confounders in diabetic adults. However, causation remains to be demonstrated.
\end{abstract}

Keywords: Monocytes, Diabetic retinopathy, Leukocyte characteristics, Cardiovascular and cerebrovascular diseases, Diabetic kidney disease

*Correspondence: wnj486@126.com; guominghao@139.com; luyingli2008@126.com

†Heng Wan, Yan Cai and Yuying Wang contributed equally to this work ${ }^{1}$ Institute and Department of Endocrinology and Metabolism,

Shanghai Ninth People's Hospital, Shanghai Jiao Tong University School of Medicine, Shanghai 200011, China

Full list of author information is available at the end of the article

\section{Introduction}

Diabetes mellitus (DM) has become a global epidemic with serious morbidity and mortality. Recent studies suggested that of the 600 million people with DM worldwide by 2040, 400 to 500 million will live in low- and middle-income countries, and half will present with diabetic microvascular complications $[1,2]$.

(c) The Author(s) 2020. This article is licensed under a Creative Commons Attribution 4.0 International License, which permits use, sharing, adaptation, distribution and reproduction in any medium or format, as long as you give appropriate credit to the original author(s) and the source, provide a link to the Creative Commons licence, and indicate if changes were made. The images or other third party material in this article are included in the article's Creative Commons licence, unless indicated otherwise in a credit line to the material. If material is not included in the article's Creative Commons licence and your intended use is not permitted by statutory regulation or exceeds the permitted use, you will need to obtain permission directly from the copyright holder. To view a copy of this licence, visit http://creativeco mmons.org/licenses/by/4.0/. The Creative Commons Public Domain Dedication waiver (http://creativecommons.org/publicdomain/ zero/1.0/) applies to the data made available in this article, unless otherwise stated in a credit line to the data. 
Diabetic retinopathy (DR), one of the diabetic microvascular complications, remains one of the leading causes of blindness, and much of the blindness from DR is preventable with early detection and treatment [3]. Epidemiological investigations have reported that approximately 1 in 3 persons with DM has DR, and 1 in 10 has proliferative DR or diabetic macular edema $[4,5]$. However, using ophthalmologists to screen every diabetic patient for DR is not feasible because most persons diagnosed with $\mathrm{DM}$ are receiving treatment from endocrinologists, and in developing countries such as China, most endocrinologists do not have ophthalmic examination equipment such as wide-field retinal photography and optical coherence tomography [ 1 , 6]. Thus, considerable attention should be paid to the development of feasible screening for DR, especially for endocrinologists in developing countries.

Plenty of evidence has suggested that retinal inflammation plays a pivotal role in the pathogenesis of DR [7-9]. Elevated inflammatory mediator levels resulting from the accumulation of advanced glycation end products may cause persistent chronic inflammation of the diabetic retina, which would bring about leukocyte activation, adhesion to the vascular endothelium and extravasation into retinal tissues $[10,11]$. Monocytes, one of the major leukocyte subtypes, have been considered as an inflammatory biomarker [12]. Animal studies have revealed the influx of considerable perivascular monocytes into retinal tissues, and the retinal pigment epithelium serves a parallel role as a gateway for monocyte trafficking to the retina $[13,14]$. One recent human study reported that the level of monocyte chemoattractant protein-1 (MCP-1), which regulates monocyte chemotaxis and modulates inflammatory processes, was significantly increased in the plasma of patients with DR [15]. However, to the best of our knowledge, no studies have evaluated the associations of monocyte levels with DR in a large number of people.

In addition, in daily clinical practice, the measurement of routinely analyzed leukocyte characteristics, which includes monocyte level, is more common than many other inflammatory markers, such as interleukin and MCP-1, because of the costs and technical difficulties in measuring the inflammatory markers. If the hypothesis that monocyte levels are associated with the prevalence of DR is demonstrated, clinicians could conduct DR screening for diabetic patients more feasibly, and this may provide evidence to support the prevention and treatment of DR by targeting monocyte trafficking. Thus, in the present study, we aimed to investigate the associations between the level of monocytes and the prevalence of DR in adults with DM.

\section{Materials and methods}

\section{Study design and participants}

The present cross-sectional study was designed in 2018. We enrolled study participants from the registration platform of the healthcare center in seven communities in Huangpu and Pudong District, Shanghai, China, from May 2018 to August 2018. The enrolled citizens were $\geq 18$ years old and had lived in the current area for $\geq 6$ months. A total of 3277 subjects with diabetes received an examination. Participants who were missing routinely analyzed leukocyte characteristics results $(n=11)$ were excluded. We then excluded participants who had received treatment, including laser photocoagulation or the use of intravitreal anti-vascular endothelial growth factor inhibitors $(n=43)$, in the past week. Finally, the number of participants who were involved in the analyses for the association between monocyte levels and the prevalence of diabetic retinopathy was 3223 .

The study protocol conformed to the ethical guidelines of the 1975 Declaration of Helsinki as reflected in the priori approval by the Ethics Committee of Shanghai Ninth People's Hospital, Shanghai Jiao Tong University School of Medicine. Written consent was obtained from all the participants in our study.

\section{Measurements}

A questionnaire about demographics, medical history, family history and lifestyle factors was completed by the same trained personnel as in our previous studies [16, 17] during the interview. Clinical examination measurements, including height, weight and blood pressure, were performed according to the same standard protocol as before $[18,19]$. Body mass index (BMI) was calculated as the weight in kilograms divided by the height in meters squared. We defined current smoking as having smoked at least 100 cigarettes in the lifetime and smoking cigarettes currently [20].

Overnight fasting blood (at least $8 \mathrm{~h}$ of fasting) was obtained between 6:00 and 9:00 am, refrigerated immediately and sent to a central laboratory for measurement in $2 \mathrm{~h}$. Routinely analyzed leukocyte characteristics, including leukocyte, neutrophil, lymphocyte, and monocyte levels, were measured with the XS-800i (Sysmex, Japan). Fasting plasma glucose (FPG), serum creatinine, total cholesterol, triglycerides, high (HDL) and low-density lipoprotein (LDL) were detected with the Beckman Coulter AU 680 (Brea, USA). Glycated hemoglobin (HbA1c) was tested using high-performance liquid chromatography with the MQ-2000PT (Shanghai, China). Morning urine samples were collected and immediately placed in the refrigerator; the levels of urine albumin and creatinine were measured with the Beckman Coulter AU 680 
(Brea, USA), and then the urine albumin/creatinine ratio (ACR) was calculated. Participants were diagnosed with DR by a remote reading conducted by ophthalmologists using retinal fundus photography with a Topcon TRCNW400 Non-Mydriatic Retinal Camera (Oakland, USA), as before $[19,21]$.

\section{Outcome definition}

The definition of dyslipidemia was total cholesterol $\geq 6.22 \mathrm{mmol} / \mathrm{L} \quad(240 \mathrm{mg} / \mathrm{dL})$, triglycerides $\geq 2.26 \mathrm{mmol} / \mathrm{L}(200 \mathrm{mg} / \mathrm{dL}), \quad \mathrm{LDL} \geq 4.14 \mathrm{mmol} / \mathrm{L}$ $(160 \mathrm{mg} / \mathrm{dL}), \mathrm{HDL}<1.04 \mathrm{mmol} / \mathrm{L}(40 \mathrm{mg} / \mathrm{dL})$, or a selfreported previous diagnosis of hyperlipidemia, according to the modified National Cholesterol Education Program-Adult Treatment Panel III. The outcome CVD was defined as a previous diagnosis with coronary heart disease, stroke, or peripheral arterial disease and was recorded in the registration platform as before [22, 23]. The estimated glomerular filtration rate (eGFR) was calculated by the Chronic Kidney Disease Epidemiology Collaboration equation for "Asian origin". The definition of DKD was ACR $\geq 30 \mathrm{mg} / \mathrm{g}$ and/or eGFR $<60 \mathrm{~mL} /$ min per $1.73 \mathrm{~m}^{2}$, as suggested by the American Diabetes Association statement [24].

The DR classification was as follows: DR stage 0 was defined as no abnormalities; DR stage 1 to 3 nonproliferative DR (NPDR) was defined as intraretinal microaneurysms, hemorrhages, venous beading, prominent microvascular abnormalities; and DR stage 4 proliferative DR (PDR) was defined as neovascularization or vitreous/ preretinal hemorrhages in accordance with the "Global Diabetic Retinopathy Project Group" [25].

\section{Statistical analysis}

IBM SPSS Statistics, Version 22 (IBM Corporation, Armonk, NY, USA) was used in the current analysis. A $P$ value (two sided) $<0.05$ indicated significance. Continuous variables were expressed as the mean $\pm S D$, and categorical variables were expressed as percentages (\%) or medians (interquartile range). One-way ANOVA or Student t-test and the Chi square test were used for the comparison of continuous and categorical variables, respectively. Monocyte levels were divided into quartiles.

A regression analysis was used to detect the associations between routinely analyzed leukocyte characteristic levels and diabetic complications. Data were summarized as odds ratios or regression coefficients ( $95 \% \mathrm{CIs}$ ). The associations of monocyte, leukocyte, neutrophil and lymphocyte levels with the prevalence of DR, CVD and DKD were tested by binary logistic regression analyses. The cutoff value of the monocyte level with the largest Youden index for predicting DR was tested by receiver operating characteristic (ROC) curve analysis.
Sensitivity analyses were performed. To minimize the impact of sample selection, the associations between the monocyte level quartiles and the prevalence of DR among the participants without PDR were evaluated in Additional file 1: Table S1. In addition, to reduce the impact of the exclusion criteria on the associations, we also investigated the associations between the monocyte level quartiles and the prevalence of DR among all 3266 participants who had reliable and complete medical records in Additional file 1: Table S2. The associations between the $\mathrm{C}$-reactive protein (CRP) level and diabetic complications are shown in Additional file 1: Table S3.

\section{Results}

The general and sociodemographic characteristics of the participants in the study are shown in Table 1. A total of 3223 diabetic participants with a mean age of 67 years old (SD 8, min 23, max 99) were involved in the final analyses. A total of 2709 (84.1\%) participants were diagnosed without DR; $334(10.4 \%)$ participants were diagnosed with DR stage 1; 166 (5.2\%) participants were diagnosed with DR stage 2; 12 (0.4\%) participants were diagnosed with DR stage 3; and $2(0.1 \%)$ participants were diagnosed with DR stage 4 . The participants were divided into two groups based on whether they had DR. Compared with the participants without DR, the duration of diabetes, FPG, HbA1c, ACR, systolic blood pressure level, and the prevalence of DKD and CKD were significantly higher in patients with DR (all $P<0.05)$. Although no differences in leukocyte, lymphocyte and neutrophil levels were found between the two groups, the monocyte level was significantly lower in patients with DR than in participants without DR $(P<0.05)$.

The characteristics of the participants by the monocyte level quartiles are shown in Table 2. Compared with the participants in the lowest monocyte level quartile, those in the highest quartile were more likely to have an older age; be male and a current smoker; have higher BMI, FPG, HbA1c, triglycerides, urine ACR, diastolic blood pressure and prevalence of dyslipidemia; and have lower total cholesterol, LDL, HDL and eGFR (all $P$ for trend $<0.05$ ). Although the prevalence of CVD and DKD among the participants in the highest monocyte level quartile were marginally and significantly higher than those in the lowest quartile, respectively, the prevalence of DR among the participants in the highest monocyte level quartile was marginally lower without adjusting for any potential confounders.

We evaluated the associations between monocyte levels and diabetic complications in Fig. 1 and found that elevated monocyte levels were significantly associated with a decreased prevalence of DR after adjusting for potential confounders; however, no significant 


\begin{tabular}{|c|c|c|c|}
\hline Characteristic & DR- & DR+ & $P$ \\
\hline$N$ & 2709 & 514 & - \\
\hline Age, years & $67.11 \pm 8.48$ & $66.96 \pm 7.92$ & 0.710 \\
\hline Men, $\%$ & 46.1 & 47.5 & 0.559 \\
\hline Duration of diabetes, years & $8(3,15)$ & $10(5,18)$ & $<0.001$ \\
\hline Current smoking, \% & 17.6 & 16.4 & 0.518 \\
\hline $\begin{array}{l}\text { Beyond high school educa- } \\
\text { tion, } \%\end{array}$ & 52.1 & 53.4 & 0.577 \\
\hline $\mathrm{BMI}, \mathrm{kg} / \mathrm{m}^{2}$ & $24.89 \pm 3.59$ & $25.29 \pm 3.63$ & 0.019 \\
\hline $\mathrm{FPG}, \mathrm{mmol} / \mathrm{L}$ & $7.73 \pm 2.34$ & $8.25 \pm 2.84$ & $<0.001$ \\
\hline $\mathrm{HbA} 1 \mathrm{c}, \%$ & $7.42 \pm 1.33$ & $7.78 \pm 1.53$ & $<0.001$ \\
\hline Total cholesterol, mmol/L & $5.12 \pm 1.19$ & $5.17 \pm 1.21$ & 0.343 \\
\hline Triglycerides, mmol/L & $1.56(1.11,2.24)$ & $1.43(1.08,2.09)$ & 0.498 \\
\hline $\mathrm{HDL}, \mathrm{mmol} / \mathrm{L}$ & $1.21 \pm 0.3$ & $1.20 \pm 0.29$ & 0.766 \\
\hline $\mathrm{LDL}, \mathrm{mmol} / \mathrm{L}$ & $3.16 \pm 0.84$ & $3.22 \pm 0.86$ & 0.176 \\
\hline $\mathrm{ACR}, \mathrm{mg} / \mathrm{g}$ & $12.0(7.0,26.0)$ & $16.0(9.0,42.5)$ & $<0.001$ \\
\hline eGFR, mL/min per $1.73 \mathrm{~m}^{2}$ & $91.56 \pm 16.90$ & $92.69 \pm 16.76$ & 0.161 \\
\hline $\begin{array}{l}\text { Systolic blood pressure, } \\
\mathrm{mmHg}\end{array}$ & $144.11 \pm 19.51$ & $148.40 \pm 20.30$ & $<0.001$ \\
\hline $\begin{array}{l}\text { Diastolic blood pressure, } \\
\mathrm{mmHg}\end{array}$ & $78.63 \pm 10.88$ & $78.63 \pm 10.80$ & 0.999 \\
\hline $\mathrm{DKD}, \%$ & 24.7 & 34.9 & $<0.001$ \\
\hline$C V D, \%$ & 36.1 & 41.0 & 0.035 \\
\hline Dyslipidemia, \% & 62.9 & 62.8 & 0.866 \\
\hline Leukocyte $\left(\times 10^{9} / \mathrm{L}\right)$ & $6.44 \pm 1.68$ & $6.38 \pm 1.62$ & 0.480 \\
\hline Lymphocytes $\left(\times 10^{9} / \mathrm{L}\right)$ & $2.09 \pm 0.65$ & $2.09 \pm 0.80$ & 0.923 \\
\hline Neutrophils $\left(\times 10^{9} / \mathrm{L}\right)$ & $3.77 \pm 1.28$ & $3.74 \pm 1.17$ & 0.608 \\
\hline Monocytes $\left(\times 10^{9} / \mathrm{L}\right)$ & $0.38 \pm 0.12$ & $0.36 \pm 0.11$ & 0.013 \\
\hline insulin therapy, \% & 15.8 & 26.6 & $<0.001$ \\
\hline
\end{tabular}

The data are summarized as the mean \pm SD for continuous variables with a distribution, the median (interquartile ranges) for continuous variables with a skewed distribution or a numerical proportion for categorical variables CVD cardiovascular and cerebrovascular disease, DKD diabetic kidney disease, $D R$ diabetic retinopathy, $B M I$ body mass index, FPG fasting plasma glucose, $H b A 1 c$ glycated hemoglobin, $H D L$ high-density lipoprotein, $L D L$ low-density lipoprotein, $A C R$ albumin to creatinine ratio, eGFR estimated glomerular infiltration rate

associations were found between monocyte levels and the prevalence of CVD and DKD after full adjustment for potential confounders. Compared with the first quartile of the monocyte level, the odds of having DR was significantly decreased by $27 \%$ for participants in the highest quartile after adjusting for age, sex and duration of diabetes $(P$ for trend $<0.01)$. One SD increase in monocyte levels was also significantly related to the prevalence of DR (OR 0.84; 95\% CI 0.75, 0.94) $(P<0.05)$. After adjusting for age, sex, education status, duration of diabetes, current smoking, BMI, HbA1c, dyslipidemia, systolic blood pressure and insulin therapy, the associations remained. The prevalence of DR among the participants in the highest quartile of peripheral blood monocyte levels significantly decreased by $41 \%$ (OR $0.59 ; 95 \%$ CI $0.43,0.81$ ) compared with the participants in the first quartile in the fullyadjusted model. Although the elevated monocyte level was significantly associated with an increased prevalence of DKD after adjusting for age, sex and duration of diabetes $(P$ for tend $<0.001)$, no association was found between the monocyte level and DKD after adjusting for age, sex, education status, duration of diabetes, current smoking, BMI, HbA1c, dyslipidemia, systolic blood pressure and insulin therapy. Furthermore, we also found no associations between the monocyte level and the prevalence of CVD in either the initial model adjusting for age, sex and duration of diabetes or in the full model adjusting for age, sex, education status, duration of diabetes, current smoking, BMI, HbA1c, dyslipidemia, systolic blood pressure and insulin therapy.

In addition, associations between leukocyte, neutrophil and lymphocyte levels and DR were detected. Figure 2 shows that the prevalence of DR was not associated with the leukocyte, neutrophil and lymphocyte levels. In the initial model adjusting for age, sex and duration of diabetes, associations of the prevalence of DR with the leukocyte, neutrophil and lymphocyte level quartiles were not found (all $P$ for trend $>0.05$ ). After adjusting for age, sex, education status, duration of diabetes history, current smoking, BMI, HbA1c, dyslipidemia, systolic blood pressure and insulin therapy, there were also no associations between the leukocyte, neutrophil and lymphocyte levels and the prevalence of DR (all $P$ for trend $>0.05$ ). The monocyte level cutoff value with the largest Youden index of was $0.405(\times 109 / \mathrm{L})$, with a sensitivity of $36.4 \%$ and specificity of $70.5 \%(P<0.05)$.

Sensitivity analyses were performed. Among the participants without proliferation, an elevated monocyte level was significantly associated with a decreased prevalence of DR in both the initial model adjusting for age, sex and duration of diabetes and in the full model adjusting for age, sex, education status, duration of diabetes, current smoking, BMI, HbA1c, dyslipidemia, systolic blood pressure and insulin therapy (Additional file 1: Table S1). In addition, among all 3266 participants who had reliable and complete medical records, the association between the monocyte level and the prevalence of DR remained (Additional file 1: Table S2). CRP was marginally associated with the prevalence of DKD. Significant associations of CRP with CVD, ln ACR, eGFR or DR were not found (Additional file 1: Table S3).

\section{Discussion}

In the present study, the results provide evidence about the unique association between monocyte levels and DR. Decreased monocyte levels were associated with 
Table 2 Characteristics of the participants by monocyte level quartiles

\begin{tabular}{|c|c|c|c|c|c|}
\hline \multirow[t]{2}{*}{ Characteristic } & \multicolumn{4}{|c|}{ Monocytes (x 109/L) } & \multirow[t]{2}{*}{$P$ for trend } \\
\hline & $\begin{array}{l}\text { Quartile } 1 \\
(\leq 0.29)\end{array}$ & $\begin{array}{l}\text { Quartile } 2 \\
(>0.29, \leq 0.36)\end{array}$ & $\begin{array}{l}\text { Quartile } 3 \\
(>0.36, \leq 0.44)\end{array}$ & $\begin{array}{l}\text { Quartile } 4 \\
(>0.44)\end{array}$ & \\
\hline N & 818 & 833 & 772 & 800 & - \\
\hline Age, years & $66.36 \pm 8.08$ & $67.14 \pm 8.02$ & $67.60 \pm 8.59$ & $67.29 \pm 8.85$ & 0.014 \\
\hline Men, \% & 28.1 & 38.5 & 54.0 & 65.5 & $<0.001$ \\
\hline Duration of diabetes, years & $8(3,15)$ & $9(3,15)$ & $10(4,16)$ & $10(4,15)$ & 0.105 \\
\hline Current smoking, $\%$ & 8.6 & 11.7 & 18.6 & 31.0 & $<0.001$ \\
\hline Beyond high school education, \% & 49.3 & 53.2 & 54.4 & 52.5 & 0.172 \\
\hline $\mathrm{BMI}, \mathrm{kg} / \mathrm{m}^{2}$ & $24.43 \pm 3.78$ & $24.75 \pm 3.47$ & $25.24 \pm 3.47$ & $25.44 \pm 3.59$ & $<0.001$ \\
\hline $\mathrm{FPG}, \mathrm{mmol} / \mathrm{L}$ & $7.46 \pm 2.33$ & $7.76 \pm 2.39$ & $8.02 \pm 2.56$ & $8.02 \pm 2.44$ & $<0.001$ \\
\hline $\mathrm{HbA} 1 \mathrm{c}, \%$ & $7.16 \pm 1.25$ & $7.41 \pm 1.33$ & $7.65 \pm 1.44$ & $7.70 \pm 1.39$ & $<0.001$ \\
\hline Total cholesterol, mmol/L & $5.27 \pm 1.26$ & $5.11 \pm 1.16$ & $5.13 \pm 1.15$ & $4.99 \pm 1.18$ & $<0.001$ \\
\hline Triglycerides, $\mathrm{mmol} / \mathrm{L}$ & $1.39(1.00,1.94)$ & $1.46(1.06,2.18)$ & $1.66(1.17,2.40)$ & $1.65(1.21,2.32)$ & $<0.001$ \\
\hline $\mathrm{HDL}, \mathrm{mmol} / \mathrm{L}$ & $1.30 \pm 0.32$ & $1.23 \pm 0.29$ & $1.18 \pm 0.28$ & $1.12 \pm 0.27$ & $<0.001$ \\
\hline $\mathrm{LDL}, \mathrm{mmol} / \mathrm{L}$ & $3.24 \pm 0.88$ & $3.16 \pm 0.85$ & $3.19 \pm 0.81$ & $3.10 \pm 0.82$ & 0.002 \\
\hline$A C R, m g / g$ & $11(7,22)$ & $12(7,28)$ & $13(7,32)$ & $15(8,32)$ & 0.026 \\
\hline eGFR, mL/min per $1.73 \mathrm{~m}^{2}$ & $93.20 \pm 15.96$ & $93.25 \pm 15.10$ & $90.73 \pm 16.96$ & $89.64 \pm 19.08$ & $<0.001$ \\
\hline Systolic blood pressure, $\mathrm{mmHg}$ & $144.01 \pm 20.60$ & $145.00 \pm 19.38$ & $145.39 \pm 19.64$ & $144.86 \pm 19.14$ & 0.352 \\
\hline Diastolic blood pressure, $\mathrm{mmHg}$ & $77.24 \pm 10.05$ & $78.01 \pm 10.79$ & $79.55 \pm 11.16$ & $79.81 \pm 11.28$ & $<0.001$ \\
\hline Dyslipidemia, \% & 54.6 & 63.3 & 65.9 & 67.9 & $<0.001$ \\
\hline$C V D, \%$ & 34.6 & 35.9 & 38.5 & 38.6 & 0.056 \\
\hline $\mathrm{DKD}, \%$ & 20.4 & 25.9 & 28.7 & 30.6 & $<0.001$ \\
\hline $\mathrm{DR}, \%$ & 16.9 & 16.7 & 16.8 & 13.4 & 0.072 \\
\hline Insulin therapy, \% & 13.2 & 15.8 & 20.3 & 21.5 & $<0.001$ \\
\hline
\end{tabular}

The data are summarized as the mean \pm SD for continuous variables with a normal distribution, the median (interquartile ranges) for continuous variables with a skewed distribution, or a numerical proportion for categorical variables

$P$ for trend was calculated by regression tests

$B M I$ body mass index, FPG fasting plasma glucose, $H b A 1 c$ glycated hemoglobin, $H D L$ high-density lipoprotein, $L D L$ low-density lipoprotein, $C V D$ cardiovascular and cerebrovascular disease, $D K D$ diabetic kidney disease, $D R$ diabetic retinopathy, $A C R$ albumin to creatinine ratio, eGFR estimated glomerular infiltration rate

increased odds of DR; however, we did not find significant associations between monocyte levels and the prevalence of CVD and DKD or between leukocyte, neutrophil and lymphocyte levels and DR after correction for potential baseline confounders. To the best of our knowledge, previous studies on the association between monocyte levels and DR are almost all basic science studies. Our study is the first large-scale population study about the association between peripheral blood monocyte levels and DR in humans, and the results imply that low peripheral blood monocyte levels may be a biomarker for screening the early stage of DR and could optimize communication between related basic science and clinical studies.

Retinal capillary occlusions, resulting from microvascular thrombi in which leucocytes play a role, have been considered as a characteristic pathologic feature in early DR [26, 27]. In addition, accumulating evidence has suggested that retinal chronic inflammation plays a dominant role in the development of DR [7-9]. Elevated levels of monocytes and neutrophils, caused by the breakdown of the blood-retinal barrier, have been found in the retinal vessels of animals and people with diabetes $[10,14,27,28]$, leading to increased retinal vascular permeability and capillary nonperfusion [13]. Studies have observed activated monocytes, which differentiate into macrophages that secrete cytokines and growth factors and interleukins, adhering to the outer surface of retinal capillaries, resulting in the breakdown of the bloodretinal barrier; the retinal pigment epithelium plays a parallel role, serving as a gateway for monocyte trafficking to the retina following direct or remote injury [13, 14, 29]. The attraction and influx of monocytes into the retina may decrease the monocyte level in the peripheral blood, accounting for our study results, which showed that decreased monocyte levels were associated with increased odds of DR. However, we did not find an association between the level of neutrophils and lymphocytes 


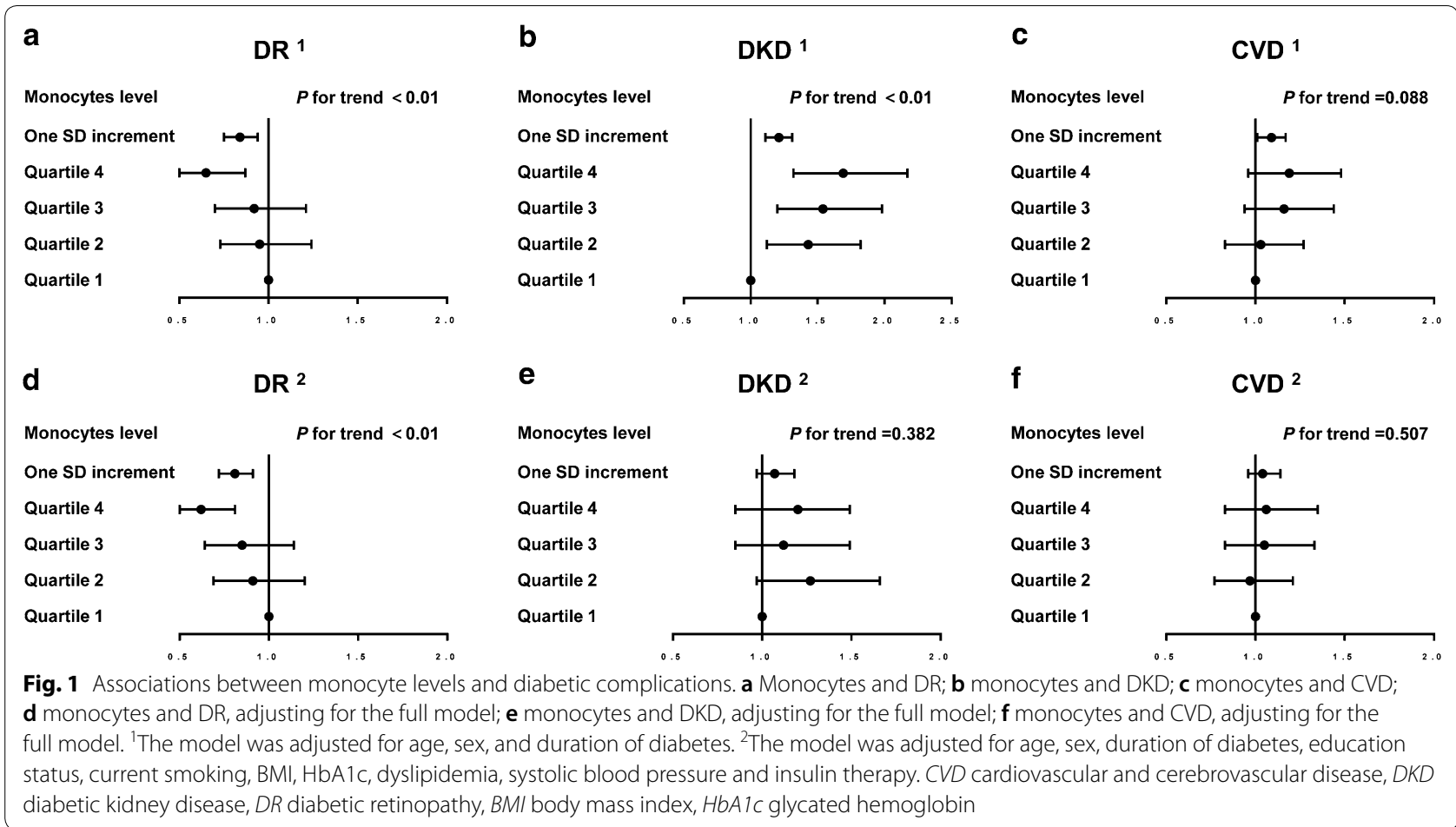

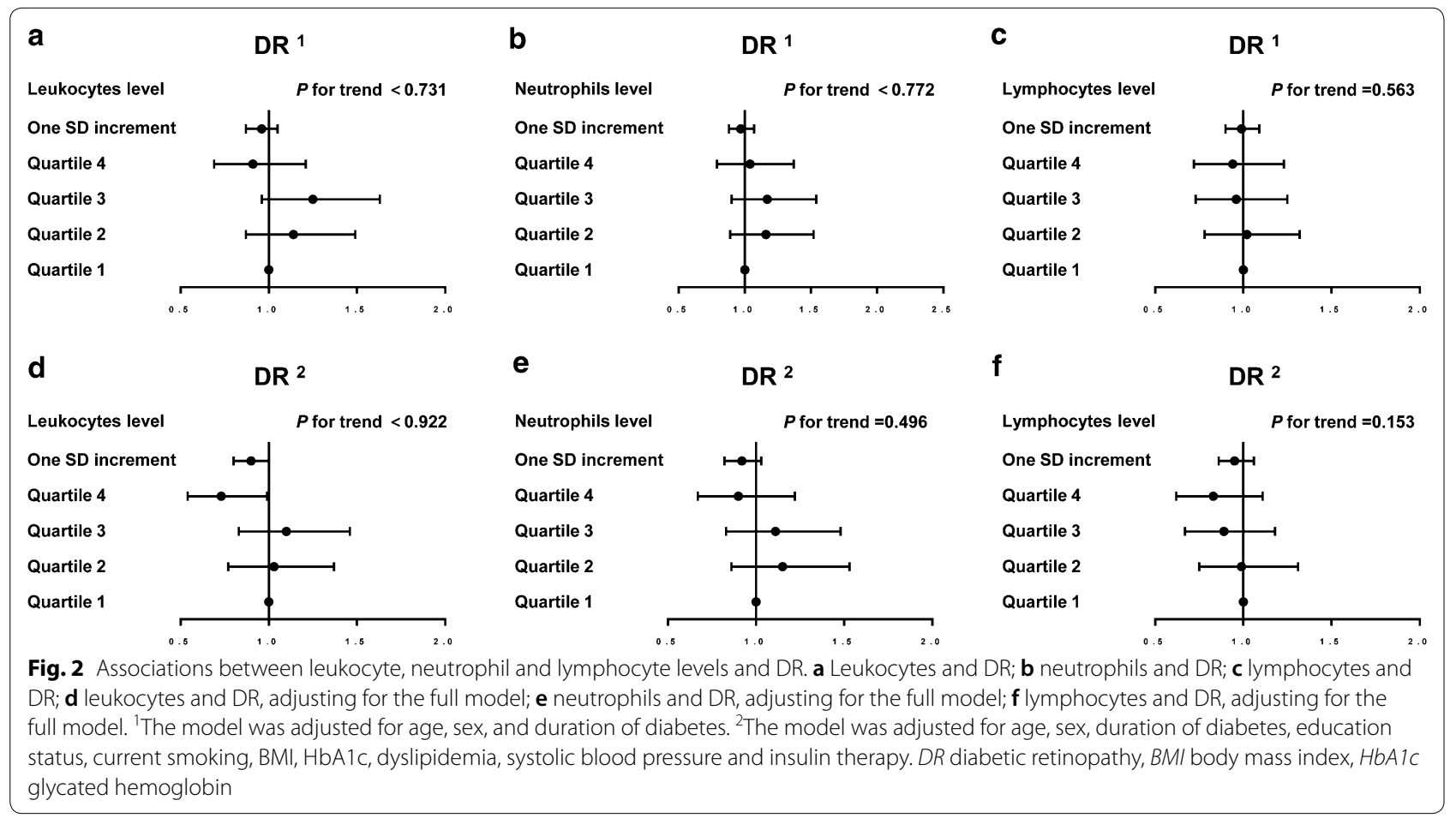

in the peripheral blood with $\mathrm{DR}$, possibly because there is a complex leukocyte life cycle in humans, where the level of leukocytes in the peripheral blood reaches a balance between the formation of leukocytes, their release from the bone marrow, and their elimination by or recruitment into the tissues [30]. It is worth noting that circulating 
monocytes are the main cell type entrapped in retinal vessels in patients with DM [31]. The difference between monocyte, neutrophil and lymphocyte cycles across the retina may account for the different associations between monocyte, neutrophil and lymphocyte levels in the peripheral blood and the prevalence of DR.

Interestingly, in the present study, there was no association between monocyte levels and the prevalence of CVD and DKD after adjusting for potential baseline confounders, although increased monocyte levels seemed to be associated with increased odds of CVD and DKD without correction. The reason may be that elevated glucose and obesity, as confounders, could promote the proliferation and activation of monocytes and macrophages with the ability to injure vascular endothelial function in diabetic patients [32-34], which is consistent with our findings. We observed that increased monocyte levels were associated with elevated FPG, HbA1c and BMI, as shown in Table 2. Thus, our findings indicate that $\mathrm{HbA1c}$ and BMI may affect the association between monocyte levels and the prevalence of CKD and DKD; however, the association between monocyte levels and the prevalence of DR was independent of HbA1c and BMI, which implies that the association between monocytes and DR is unique and provides evidence for a therapeutic strategy targeting monocyte trafficking for treating DR. The mechanisms explaining the opposite trend in the different diabetic complications are still not clear. We suspect there are two possible reasons. First, different chemotactic responses of monocytes to different organs may account for the different associations between monocyte levels and the prevalence of CKD, DKD and DR [35, 36]. We suspected that the chemotactic responses of monocytes to capillaries of the retina may be stronger than those of heart arteries. Blunted chemotactic responses of monocytes to cardiac arteriogenic stimuli in individuals with DM compared to those without DM, leading to impaired cardiac arteriogenesis [36], increases the susceptibility of diabetic individuals to cardiac events with respect to elevated cardiovascular mortality [37]. However, neovascularization resulting from monocyte aggregation in retinal capillaries is the primary manifestation of PDR, which can lead to acquired blindness [35]. Second, the attraction and influx of monocytes into the retina by adhering to the outer surface of retinal capillaries and breaking down the blood-retinal barrier may decrease the monocyte level in the peripheral blood [13, 14].

In fact, to the best of our knowledge, only one casecontrol study with 246 patients diagnosed with T2DM, 121 of whom had DR (62 people had NPDR and 59 people had PDR), reported that no significant association was found between monocyte level and DR [38]. The difference in sample size may account for the different results. In addition, to minimize the impact of sample selection, we evaluated the associations between monocyte levels and DR among the participants without proliferative DR (Additional file 1: Table S1) and among all 3266 participants (Additional file 1: Table S2) without excluding the participants treated with laser photocoagulation or intravitreal anti-vascular endothelial growth factor inhibitors, and the results remained consistent.

Although it was an investigation of a large sample of community dwelling participants with strong quality control, there were some limitations in the present study. First, this was a cross-sectional study; thus, causal relationships between monocyte levels and DR cannot be confirmed. The present findings should be cautiously interpreted, and further prospective studies are needed. Second, the prevalence of proliferative DR was low among the participants enrolled in the study, which restricted the extrapolation of the current results to all the stages of DR. Third, although the large sample size of this study has advantages in enhancing the reliability of our findings, a statistically significant finding might not necessarily be clinically significant because of the large sample size in this study; thus, significant findings derived from this study must be further analyzed and interpreted with caution.

\section{Conclusions}

We observed that the decreased level of peripheral blood monocytes was associated with increased odds of DR after adjusting for potential confounders in Chinese adults with DM, which suggests that measuring monocyte levels in a timely manner may be helpful to screen for the early stage of DR. However, the causation between monocytes and DR should be assessed in additional cohort studies.

\section{Supplementary information}

Supplementary information accompanies this paper at https://doi. org/10.1186/s12967-020-02422-9.

Additional file 1: Table S1. Associations between the monocytes level quartiles and the prevalence of DR among the participants without proliferative DR. Table S2. Associations between the monocytes level quartiles and the prevalence of DR among all the 3266 participants. Table S3.

Associations between CRP level and diabetic complications.

\section{Abbreviations}

DM: Diabetes mellitus; DR: Diabetic retinopathy; PDR: Proliferative diabetic retinopathy; NPDR: Nonproliferative diabetic retinopathy; BMI: Body mass index; FPG: Fasting plasma glucose; HbA1c: Glycated hemoglobin; HDL: High-density lipoprotein; LDL: Low-density lipoprotein; CVD: Cardiovascular and cerebrovascular diseases; ACR: Albumin to creatinine ratio; DKD: Diabetic kidney disease; eGFR: Estimated glomerular infiltration rate; MCP-1: Monocyte chemoattractant protein-1; ROC: Receiver operating characteristic; CRP:

C-reactive protein level; OR: Odds ratios; Cls: Confidence intervals. 


\author{
Acknowledgements \\ The authors thank all team members and participants in the study.
}

\section{Authors' contributions}

YL and NW designed the study; HW, YC, YW, SF, CC, YC, FX and MG conducted the research; $H W, Y C$ and $Y W$ analyzed the data; $H W$ wrote the original draft; MG and HW wrote the revised manuscript. All authors read and approved the final manuscript.

\section{Funding}

This study was supported by National Natural Science Foundation of China (91857117, 81600614); Yunnan Province Lu Yingli Expert Workstation; Science and Technology Commission of Shanghai Municipality (19140902400, 18410722300); the Major Science and Technology Innovation Program of Shanghai Municipal Education Commission (2019-01-07-00-01-E00059); Commission of Health and Family Planning of Pudong District (PWZxq2017-17); Municipal Human Resources Development Program for Outstanding Young Talents in Medical and Health Sciences in Shanghai (2017YQ053); Shanghai JiaoTong University School of Medicine (19XJ11007). The funders played no role in the design or conduct of the study, collection, management, analysis, or interpretation of data or in the preparation, review, or approval of the article.

\section{Availability of data and materials}

The data supporting the findings of this study are available on reasonable request from the corresponding authors.

\section{Ethics approval and consent to participate}

The study protocol was approved by the Ethics Committee of Shanghai Ninth People's Hospital, Shanghai Jiao Tong University School of Medicine. The study protocol conformed to the ethical guidelines of the 1975 Declaration of Helsinki as reflected in a priori approval by the appropriate institutional review committee. Informed consent was obtained from all participants included in the study.

\section{Consent for publication}

Not applicable.

\section{Competing interests}

The authors declare that they have no competing or financial interests regarding to the submitted work.

\section{Author details}

${ }^{1}$ Institute and Department of Endocrinology and Metabolism, Shanghai Ninth People's Hospital, Shanghai Jiao Tong University School of Medicine, Shanghai 200011, China. ${ }^{2}$ Department of Endocrinology, The Fifth Affiliated Hospital of Kunming Medical University, Yunnan Honghe Prefecture Central Hospital (Ge Jiu People's Hospital), Yunnan, China. ${ }^{3}$ Department of Ophthalmology, Shanghai Ninth People's Hospital, Shanghai Jiao Tong University School of Medicine, Shanghai, China.

Received: 15 April 2020 Accepted: 17 June 2020

Published online: 22 June 2020

\section{References}

1. Wong TY, Sun J, Kawasaki R, Ruamviboonsuk P, Gupta N, Lansingh VC, Maia M, Mathenge W, Moreker S, Muqit MMK, et al. Guidelines on diabetic eye care: the international council of ophthalmology recommendations for screening, follow-up, referral, and treatment based on resource settings. Ophthalmology. 2018;125:1608-22.

2. Zheng Y, Ley SH, Hu FB. Global aetiology and epidemiology of type 2 diabetes mellitus and its complications. Nat Rev Endocrinol. 2018;14:88-98.

3. Liew G, Lei Z, Tan G, Joachim N, Ho IV, Wong TY, Mitchell P, Gopinath B, Crossett B. Metabolomics of diabetic retinopathy. Curr Diab Rep. 2017;17:102.

4. Ting DS, Cheung GC, Wong TY. Diabetic retinopathy: global prevalence, major risk factors, screening practices and public health challenges: a review. Clin Exp Ophthalmol. 2016;44:260-77.
5. Yau JW, Rogers SL, Kawasaki R, Lamoureux EL, Kowalski JW, BekT, Chen SJ, Dekker JM, Fletcher A, Grauslund J, et al. Global prevalence and major risk factors of diabetic retinopathy. Diabetes Care. 2012;35:556-64.

6. Horton MB, Silva PS, Cavallerano JD, Aiello LP. Operational components of telemedicine programs for diabetic retinopathy. Curr Diab Rep. 2016;16:128.

7. Youngblood H, Robinson R, Sharma A, Sharma S. Proteomic biomarkers of retinal inflammation in diabetic retinopathy. Int J Mol Sci. 2019;20:4755.

8. Willermain F, Scifo L, Weber C, Caspers L, Perret J, Delporte C. Potential interplay between hyperosmolarity and inflammation on retinal pigmented epithelium in pathogenesis of diabetic retinopathy. Int J Mol Sci. 2018;19:1056.

9. Moschos MM, Pantazis P, Gatzioufas Z, Panos GD, Gazouli M, Nitoda E, Brouzas D. Association between platelet activating factor acetylhydrolase and diabetic retinopathy: Does inflammation affect the retinal status? Prostaglandins Other Lipid Mediat. 2016;122:69-72.

10. Kim SY, Johnson MA, McLeod DS, Alexander T, Hansen BC, Lutty GA. Neutrophils are associated with capillary closure in spontaneously diabetic monkey retinas. Diabetes. 2005;54:1534-42.

11. Joussen AM, Murata T, Tsujikawa A, Kirchhof B, Bursell SE, Adamis AP. Leukocyte-mediated endothelial cell injury and death in the diabetic retina. Am J Pathol. 2001;158:147-52.

12. Badr RE, Salama MI, Abd-Elmaogood AK, Eldeib AEM. Toll-like receptor 2 expression on monocytes and microvascular complications in type 2 diabetic patients. Diabetes Metab Syndr. 2019;13:1299-302.

13. Benhar I, Reemst K, Kalchenko V, Schwartz M. The retinal pigment epithelium as a gateway for monocyte trafficking into the eye. EMBO $\mathrm{J}$. 2016;35:1219-35.

14. Rangasamy S, McGuire PG, Franco Nitta C, Monickaraj F, Oruganti SR, Das A. Chemokine mediated monocyte trafficking into the retina: role of inflammation in alteration of the blood-retinal barrier in diabetic retinopathy. PLOS ONE. 2014;9:e108508.

15. Jiang Z, Hennein L, Xu Y, Bao N, Coh P, Tao L. Elevated serum monocyte chemoattractant protein-1 levels and its genetic polymorphism is associated with diabetic retinopathy in Chinese patients with type 2 diabetes. Diabet Med. 2016;33:84-90.

16. Wang N, Wang X, Li Q, Han B, Chen Y, Zhu C, Chen Y, Lin D, Wang B, Jensen MD, Lu Y. The famine exposure in early life and metabolic syndrome in adulthood. Clin Nutr. 2017;36:253-9.

17. Wang N, Chen Y, Ning Z, Li Q, Han B, Zhu C, Chen Y, Xia F, Jiang B, Wang B, et al. Exposure to famine in early life and nonalcoholic fatty liver disease in adulthood. J Clin Endocrinol Metab. 2016;101:2218-25.

18. Wang Y, Wan H, Chen Y, Xia F, Zhang W, Wang C, Fang S, Zhang K, Li Q, Wang N, Lu Y. Association of C-peptide with diabetic vascular complications in type 2 diabetes. Diabetes Metab. 2020;46:33-40.

19. Wan H, Wang Y, Zhang K, Chen Y, Fang S, Zhang W, Wang C, Li Q, Xia F, Wang N, Lu Y. Associations between vitamin D and microvascular complications in middle-aged and elderly diabetic patients. Endocr Pract. 2019;25:809-16.

20. Xu Y, Wang L, He J, Bi Y, Li M, Wang T, Wang L, Jiang Y, Dai M, Lu J, et al. Prevalence and control of diabetes in Chinese adults. JAMA. 2013;310:948-59.

21. Wang C, Zhang W, Wang Y, Wan H, Chen Y, Xia F, Zhang K, Wang N, Lu Y. Novel associations between sex hormones and diabetic vascular complications in men and postmenopausal women: a cross-sectional study. Cardiovasc Diabetol. 2019;18:97.

22. Carrelli AL, Walker MD, Lowe H, McMahon DJ, RundekT, Sacco RL, Silverberg SJ. Vitamin D deficiency is associated with subclinical carotid atherosclerosis: the Northern Manhattan study. Stroke. 2011;42:2240-5.

23. Wan H, Wang Y, Chen Y, Fang S, Zhang W, Xia F, Wang N, Lu Y. Different associations between serum urate and diabetic complications in men and postmenopausal women. Diabetes Res Clin Pract. 2020;160:108005.

24. American Diabetes A. Microvascular complications and foot care: standards of medical care in diabetes-2019. Diabetes Care. 2019;42:S124-38.

25. Wilkinson CP, Ferris FL 3rd, Klein RE, Lee PP, Agardh CD, Davis M, Dills D, Kampik A, Pararajasegaram R, Verdaguer JT, Global Diabetic Retinopathy Project G. Proposed international clinical diabetic retinopathy and diabetic macular edema disease severity scales. Ophthalmology. 2003;110:1677-82. 
26. Kaplar M, Kappelmayer J, Veszpremi A, Szabo K, Udvardy M. The possible association of in vivo leukocyte-platelet heterophilic aggregate formation and the development of diabetic angiopathy. Platelets. 2001;12:419-22.

27. Schroder S, Palinski W, Schmid-Schonbein GW. Activated monocytes and granulocytes, capillary nonperfusion, and neovascularization in diabetic retinopathy. Am J Pathol. 1991;139:81-100.

28. McLeod DS, Lefer DJ, Merges C, Lutty GA. Enhanced expression of intracellular adhesion molecule-1 and P-selectin in the diabetic human retina and choroid. Am J Pathol. 1995;147:642-53.

29. Eichler W, Lohrenz A, Simon KU, Krohn S, Lange J, Burger S, Liebscher I. The role of ADGRE5/CD97 in human retinal pigment epithelial cell growth and survival. Ann NY Acad Sci. 2019;1456:64-79.

30. Hidalgo A, Chilvers ER, Summers C, Koenderman L. The neutrophil life cycle. Trends Immunol. 2019;40:584-97.

31. Chen M, Obasanmi G, Armstrong D, Lavery NJ, Kissenpfennig A, Lois N, $\mathrm{Xu} \mathrm{H}$. STAT3 activation in circulating myeloid-derived cells contributes to retinal microvascular dysfunction in diabetes. J Neuroinflammation. 2019;16:138.

32. Stunault MI, Bories G, Guinamard RR, Ivanov S. Metabolism plays a key role during macrophage activation. Mediators Inflamm. 2018;2018:2426138.

33. Torres-Castro I, Arroyo-Camarena UD, Martinez-Reyes CP, Gomez-Arauz AY, Duenas-Andrade Y, Hernandez-Ruiz J, Bejar YL, Zaga-Clavellina V, Morales-Montor J, Terrazas LI, et al. Human monocytes and macrophages undergo M1-type inflammatory polarization in response to high levels of glucose. Immunol Lett. 2016;176:81-9.
34. Tu TH, Kim CS, Nam-Goong IS, Nam CW, Kim YI, Goto T, Kawada T, Park T, Yoon Park JH, Ryoo ZY, et al. 4-1BBL signaling promotes cell proliferation through reprogramming of glucose metabolism in monocytes/macrophages. FEBS J. 2015;282:1468-80.

35. Wong TY, Cheung CM, Larsen M, Sharma S, Simo R. Diabetic retinopathy. Nat Rev Dis Primers. 2016;2:16012.

36. Tchaikovski V, Olieslagers S, Bohmer FD, Waltenberger J. Diabetes mellitus activates signal transduction pathways resulting in vascular endothelial growth factor resistance of human monocytes. Circulation. 2009;120:150-9.

37. Dorenkamp M, Muller JP, Shanmuganathan KS, Schulten H, Muller N, Loffler I, Muller UA, Wolf G, Bohmer FD, Godfrey R, Waltenberger J. Hyperglycaemia-induced methylglyoxal accumulation potentiates VEGF resistance of diabetic monocytes through the aberrant activation of tyrosine phosphatase SHP-2/SRC kinase signalling axis. Sci Rep. 2018;8:14684.

38. Yue S, Zhang J, Wu J, Teng W, Liu L, Chen L. Use of the monocyte-tolymphocyte ratio to predict diabetic retinopathy. Int J Environ Res Public Health. 2015;12:10009-19.

\section{Publisher's Note}

Springer Nature remains neutral with regard to jurisdictional claims in published maps and institutional affiliations.
Ready to submit your research? Choose BMC and benefit from:

- fast, convenient online submission

- thorough peer review by experienced researchers in your field

- rapid publication on acceptance

- support for research data, including large and complex data types

- gold Open Access which fosters wider collaboration and increased citations

- maximum visibility for your research: over 100M website views per year

At BMC, research is always in progress.

Learn more biomedcentral.com/submissions 